\title{
संगीत की गायन विधा में कंठ साधना का महत्त्व
}

DR. THAKUR SINGH

Instructor (Vocal), Department of Music, Panjab University, Chandigarh

\section{सार संक्षेपिका}

प्रस्तुत शोध पत्र संगीत की गायन विधा में कंठ साधना के महत्त्व पर केन्द्रित है। संगीत की तीन विधाओं-गायन, वादन और नृत्य में से गायन प्रमुख है। यह तीनों विधाएं स्वर द्वारा परस्पर बंधी हुई है। स्वर के उद्गम केन्द्रों में से मानव कंठ सर्वोपरी है। इसलिए गायन विधा में कंठ साधना का महत्त्वपूर्ण स्थान है। प्राचीनतम शास्त्रों में गान के गुण व दोष का उल्लेख प्राप्त होता है। कंठ साधना करते समय इन सभी गुण-दोषों का ध्यान रखते हुए अभ्यास करना चाहिए। अभ्यास करते समय आवाज का निकास, स्वर की स्थिरता व श्वास नियंत्रण का भी ध्यान रखा जाता है। इसके अतिरिक्त अभ्यास का स्थान, अभ्यास का समय, अभ्यास की अवधि एवं मानसिक तथा शारीरिक शुद्धता का ध्यान रखते हुए अभ्यास करना चाहिए। गायन का अभ्यास करते समय निम्नलिखित क्रम में कंठ साधना करनी चाहिए- षड़ज साधना, खरज़ साधना, अन्य स्वरों की साधना, अलंकार व पल्टों का अभ्यास, गमक, मींड इत्यादि सौंदर्यवर्धक तत्त्वों का अभ्यास, विभिन्न रागों तथा बंदिशों का अभ्यास इत्यादि।

बीज शब्द

संगीत, गायन विधा, कंठ साधना

भूमिका

संगीत की तीन विधाओं का क्रम इस प्रकार माना जाता है- गायन, वादन और नृत्य। इन तीनों विधाओं में से गायन प्रमुख है क्योंकि वादन, गायन का अनुसरण करता है और नृत्य, गायन व वादन दोनों का अनुसरण करता है। संगीत की यह तीन विधाएं एक अदृश्य सूत्र 'स्वर' से परस्पर बंधी हुई हैं। शास्त्रों के अध्ययन से यह ज्ञात होता है कि स्वर के मुख्य उद्गम स्थान दो हैं मानव कंठ तथा वीणा। वीणा आदि वाद्यों पर केवल स्वर का उद्गम संभव है शब्द का नहीं। परंतु मानव कंठ द्वारा स्वर के साथ-साथ भाषा या शब्द का उद्गम भी सहज रूप में होता है। कंठ के द्वारा स्वर का उद्गम उचित रूप से करने के लिए कंट को साधना पड़ता है इसलिए गायन में कंठ साधना का महत्त्वपूर्ण स्थान है। अतः कण्ठ साधना एक प्रकार की तपस्या है जिसके आधार पर गायक एक ऐसा आत्म-विश्वास अर्जित करता है जिससे वह स्वर व राग समूह का इच्छानुसार प्रस्तुतिकरण कर सके।

प्राचीनतम शास्त्रों में गायक के गुण-दोष का भली भांति चित्रण किया गया है। नारदीय शिक्षा में में मानव कंठ के 6 गुण तथा पाँच दोषों का वर्णन इस प्रकार किया गया है1-गान के 10 गुण व 14 दोषों का वर्णन है। इसी प्रकार नाट्यशास्त्र

श्रावको (णो) ऽथ घन: स्निग्धो मधुरो ह्मवधानवान्

त्रिर्थानाशोभीत्येंव तु षट् कण्ठस्य गुणा मताः

इसी प्रकार मानव कंट के 5 दोषों का इस प्रकार वर्णन किया गया है-

1 मिश्रा कान्ता प्रसाद, वॉयसकल्चर-ज्ञान एवं परंपरा (हिन्दुस्तानी म्यूज़िक), पृष्ठ-29-30 
कपिलोह्म (उव्य) वस्थितश्चैव तथा सन्दष्ट एव च।

काकी च तुम्बकी च (चवै) पन्च दौषा भवन्ति हि।

अर्थात् महर्षि भरत द्वारा गानयोग्य कंठ के श्रावक, घन, स्निग्ध, मधुर, अवधानवान् तथा त्रिस्थानशोभी नामक छह: गुण तथा कपिल, अव्यवस्थित, संदष्टक, काकी एवं तुम्बकी नामक पाँच दोष बताए गए हैं। शिक्षार्थी अथवा गायक को इन गुण व दोषों का ध्यान रखते हुए कंठ साधना का अभ्यास करना चाहिए। प्रसिद्ध विद्वान पं. भातखंडे जी ने आवाज को साधने के लिए निम्नलिखित तीन उपायों के बारे में बताया है- ${ }^{1}$

1. आवाज ठीक प्रकार से निकालना

2. आवाज की स्थिरता

\section{3. श्वास नियंत्रण।}

अर्थात् प्रथम रूप में कठ से ध्वनि का उपयुक्त निकास होना चाहिए जो कि एक संगीत गुरु ही बता सकता है। इसके पश्चात गाते समय आवाज स्थिर रहनी चाहिए। आवाज में कंपन तथा अस्थिरता एक प्रमुख कंठ दोष माना जाता है। इसके अतिरिक्त कंठ साधना करते समय श्वास नियंत्रण करने के लिए धीमी गति में अभ्यास करना चाहिए।

कंठ साधना करते समय विभिन्न वर्णों तथा नाद का उच्चारण स्पष्ट रूप में किया जाना चाहिए। वर्णों में मुख्य स्वर -अ, आ, ई, ई, उ, ऊ इत्यादि की संख्या 13 है तथा व्यंजनों की संख्या 33 है। इसके अतिरिक्त संगीतोपयोगी अक्षर-स, रे, ग, म, प, ध, नि इत्यादि का उच्चारण शुद्ध रूप में किया जाना चाहिए। इसके अतिरिक्त कंठ साधना करते समय शिक्षार्थी को अन्य बहुत-से तत्त्वों का ध्यान रखना चाहिए जैसे -

\section{अभ्यास का स्थान}

शिक्षार्थी को अभ्यास करने वाले स्थान का विशेष ध्यान रखना चाहिए। इसके लिए उचित रूप से स्वच्छ, शाँतिवर्धक स्थान होना अनिवार्य है।

\section{अभ्यास का समय}

अभ्यास करने के लिए सबसे उत्तम समय ब्रह्ममुर्हूत है। इसके पश्चात उत्तम समय सूर्यास्त होता है। इसके अतिरिक्त अन्य किसी भी सुविधापूर्वक समय पर अभ्यास किया जा सकता है।

\section{अभ्यास की अवधि}

कंट साधना के अभ्यास की अवधि को धीरे-धीरे बढ़ाया जाना चाहिए। एक ही बार में दीर्घ समय तक अभ्यास करने से आवाज का असहज व दवाबपूर्ण होना स्वाभाविक है।

1 भातखंडे विष्णु नारायण, क्रमिक पुस्तक मालिका, भाग-6, पृष्ठ-22 


\section{शारीरिक तथा मानसिक अनुशासन}

शिक्षार्थी को कंट साधना करने के पूर्व शारीरिक स्वच्छता का अवश्य ध्यान रखना चाहिए। इसके अंतर्गत शौचादि क्रियाओं से निवृत्त होना, खान-पान का ध्यान रखना तथा ब्रह्मचर्य का पालन करना इत्यादि आता है। इसके अतिरिक्त शिक्षार्थी को धैर्य, संयम, सहजता तथा निरंतरता का पालन करते हुए अभ्यास करना चाहिए।

कंठ साधना करते समय शिक्षार्थी को निम्न क्रम में अभ्यास करना चाहिए।

\section{1. षड़ज साधना}

अभ्यास में सर्वप्रथम जिस तत्त्व का सबसे अधिक ध्यान रखा जाता है-वह स्वर है षड्ज। यह सभी स्वरों का मूलाधार है। इसी से अन्य स्वरों की उत्पत्ति होती है। गुणीजन षड्ज स्वर के बारे में यह प्रमाण देते हैं कि-

एक साधे सब सधे, सब साधे सब जाए।

अर्थात् षड़ज को साधने से शेष अन्य स्वर सहजता से साधे जा सकते हैं। कंठ साधना सदैव तानपुरे पर ही की जानी चाहिए। सर्वप्रथम शिक्षार्थी को केवल तानपुरा सुनना व धीरे-धीरे षड़ज लगाव का शिक्षण ग्रहण करना चाहिए। षड़ज लगाव में सर्वप्रथम 'उम्' शब्द का उच्चारण करना चाहिए। उसके पश्चात् 'ओ३म' का उच्चारण करना चाहिए। धीरे-धीरे स्वर स्थिर होने पर अ, आ, इ, ई इत्यादि स्वरों का उच्चारण षड़ज स्वर पर करना चाहिए। इस प्रक्रिया का कोई विशेष समय निर्धारण नहीं है। प्रारंभ में शिक्षार्थी को षड़ज साधना कम-से-कम 1-2 महीने तक रोजना एक निश्चित समय पर निरंतर रूप में करनी चाहिए जिससे कि षड़ज स्थिर, सहज व मधुर रूप धारण कर सके।

\section{2. खरज साधना}

षड़ज साधना के पश्चात शिक्षार्थी को मन्द्र सप्तक के स्वरों की क्रमानुसार साधना करनी चाहिए। इसे खरज साधना भी कहा जाता है। मन्द्र सप्तक के स्वरों को साधने का क्रम नी ध प म इत्यादि अवरोही क्रम में करना चाहिए। प्रत्येक स्वर पर कम-से-कम दो-तीन लंबे श्वास तक स्वर विश्राम करना चाहिए। संगीत के विद्वानों तथा उस्तादों द्वारा अक्सर यह कहा जाता है कि मन्द्र सप्तक के स्वरों को साधने से स्वरों में स्थिरता, प्रगाढ़ता तथा सहजता का उद्गम होता है। इसलिए शिक्षार्थी को नित्य अभ्यास में खरज़ साधना अवश्य करनी चाहिए।

\section{3. अन्य स्वरों की साधना}

खरज साधना के उपरांत मध्य सप्तक के अन्य स्वरों की तरफ बढ़ा जाता है जिनमें ऋषभ, गंधार, मध्यम, पंचम, धैवत, निषाद इत्यादि स्वर आते हैं। इस साधना को करते समय विद्यार्थी को अपने गले की क्षमतानुसार स्वरों के क्रम में आगे बढ़ना चाहिए। सर्वप्रथम कंठ साधना में केवल शुद्ध स्वरों की साधना ही करनी चाहिए। मध्य सप्तक के स्वरों का अभ्यास करने के पश्चात शिक्षार्थी को 
सर्वप्रथम आधारभूत अलंकार सा रे ग म प ध नी सां, सां नी ध प म ग रे सा को मध्य लय में गायन करने का अभ्यास करना चाहिए। आवश्यकतानुसार गायन के दौरान प्रत्येक स्वर पर ताली भी लगाई जा सकती है जिससे स्वर के साथ-साथ विद्यार्थी की लय भी एकरूपता प्रदान करती है। इस साधना के दौरान शिक्षार्थी को बहुत-सी बातों का ध्यान रखते हुए अभ्यास करना चाहिए जैसे-आकार के साथ स्वर का उच्चारण करना, मुंह की बनावट, स्वर की सहजता, स्थिरता एवं मधुरता तथा लय की एकरूपता इत्यादि।

\section{4. अलंकार व पल्टों की साधना}

अलंकार का सामान्य अर्थ है-आभूषण। जिस प्रकार एक रत्री आभूषणों से अपनी सुदंरता को बढ़ावा देती है उसी प्रकार एक संगीतज्ञ अलंकारों से राग को सजाता है। कंठ साधना में अलंकारों का विशेष योगदान रहता है। पल्टे, अलंकार का ही रूप होते हैं जिसमें स्वरों का उलट-पलट कर प्रयोग किया जाता है। शिक्षार्थी को अलंकार तथा पल्टों की साधना अवश्य नियमित रूप से करनी चाहिए। इससे विद्यार्थी का स्वर के प्रति नियंत्रण तथा गले की तैयारी में काफी लाभ होता है। शास्त्रों में विभिन्न अलंकारों के साथ 'खण्डमेरू' नामक अलंकारों के अभ्यास का भी प्रचलन है। नीचे कुछ जटिल अलंकारों के उदाहरण इस प्रकार हैं-

(क) आरोह - स रे ग स रे ग स रे स ग रे स, रे ग म रे ग म रे ग रे म ग रे, ग अवरोह - $\quad$ सं नी ध सं नी ध सं नी सं ध नी सं, नी ध प नी ध प नी ध प नी ध नी प ध नी, ध - - - -

(ख) आरोह - स रे ग रे रे स, रे ग रे ग रे रे रे स, रे ग रे ग स रे स ग रे ग, रे ग ग स रे स रे ग) रे - - - अवरोह - सं नी ध नी नी सं, नी ध नी ध नी नी नी सं, नी ध नी ध सं नी सं ध नी ध, नी ध ध सं नी सं नी ध) नी - - 5. गमक, मींड, खटका आदि सौंदर्यवर्धक तत्वों का अभ्यास अलंकार व पल्टों के पश्चात् शिक्षार्थी को गमक, खटका इत्यादि का अभ्यास करना चाहिए। इस अभ्यास को सर्वप्रथम गुरु के सम्मुख ही करना चाहिए। इसके पश्चात ही इसे एकल अभ्यास के रूप में प्रयोग में लाना चाहिए। इसके अतिरिक्त अन्य सौंदर्यवर्धक तत्वों में मींड, कण, मुर्की इत्यादि का अभ्यास पूर्व शिक्षण के पश्चात किया जाना चाहिए। 


\section{6. विभिन्न रागों व बंदिशों का अभ्यास}

स्वर साधना के पश्चात शिक्षार्थी को आधारभूत रागों जैसे-बिलावल, भूपाली, यमन, भैरव, दुर्गा इत्यादि रागों का शिक्षण ग्रहण करने के पश्चात उसका अभ्यास करना चाहिए। राग का अभ्यास करने के लिए सर्वप्रथम राग के मूलभूत चलन या आरोह-अवरोह का अभ्यास करना चाहिए जिससे राग के स्वरों पर नियंत्रण हो सके। इसके साथ-साथ राग के विशेष रागानुरूप पलटों का अभ्यास भी किया जा सकता है जैसे राग यमन में एक पल्टे का विवरण नीचे दिया जा रहा है-

\section{नी रे ग मे प मे ग रे, ग मे ध नी सं नी ध प, मे ध नी रें गं रें सं नी ध प मे ग रे स नी स}

इस प्रकार शेष अन्य रागों में भी विशेष पल्टों के अभ्यास द्वारा राग के स्वरों पर नियंत्रण बनाया जा सकता है। इसके पश्चात रागों में आधारभूत बंदिशों का अभ्यास किया जाना चाहिए। इसके साथ-साथ राग में सरगमगीत, लक्षणगीत इत्यादि का भी अभ्यास किया जा सकता है। सरगमगीत व लक्षणगीत का अभ्यास करने से राग का चलन, वादी-संवादी इत्यादि राग लक्षणों से शिक्षार्थी भली-भांति परिचित हो जाता है। अतः हम देखते हैं कि उपर्युक्त प्रणाली का अनुसरण करते हुए शिक्षार्थी अपनी कंठ साधना के क्षेत्र में अधिक-से-अधिक लाभ प्राप्त कर सकता है।

सन्दर्भ

भातखंडे, विष्णु नारायण (2015), क्रमिक पुस्तक मालिका, भाग-6, पृष्ठ-22, संगीत कार्यालय, हाथरस, उ. प्र.

मिश्रा, कान्ता प्रसाद (2011), वॉयसकल्चर-ज्ञान एवं परंपरा (हिन्दुस्तानी म्यूज़िक), कनिष्क पब्लिशर्स, डिस्ट्रीब्यूटर्स, नई दिल्ली

यमन, अशोक कुमार (2015), संगीत रत्नावली, अभिषेक पब्लिकेशन्स, चण्डीगढ़। 